\title{
Prognostic role of beclin-1 in locally advanced non-small cell lung cancer in patients receiving docetaxel-platinum induction chemotherapy
}

\author{
Hee Yeon Lee ${ }^{1}$, Jung Ha Shin ${ }^{2}$, Kyo-Young Lee ${ }^{3}$, Jae Kil Park ${ }^{4}$, Sook Whan Sung ${ }^{4}$, Yeon Sil Kim \\ Jin-Hyoung Kang ${ }^{6}$, and Jeong-Oh $\mathrm{Kim}^{7}$
}

\begin{abstract}
${ }^{1}$ Department of Internal Medicine, College of Medicine, Incheon St. Mary's Hospital, The Catholic University of Korea, Incheon; ${ }^{2}$ Department of Pathology, College of Medicine, St. Paul's Hospital, The Catholic University of Korea, Seoul; Departments of ${ }^{3}$ Pathology, ${ }^{4}$ Thoracic and Cardiovascular Surgery, ${ }^{5}$ Radiation Oncology, and ${ }^{6}$ Internal Medicine, College of Medicine, Seoul St. Mary's Hospital, The Catholic University of Korea, Seoul; ${ }^{7}$ Laboratory of Medical Oncology, Cancer Research Institute, College of Medicine, The Catholic University of Korea, Seoul, Korea
\end{abstract}

Received: January 6, 2017

Revised : July 11, 2017

Accepted: July 19, 2017

\section{Correspondence to}

Jin-Hyoung Kang, M.D.

Department of Internal

Medicine, College of Medicine,

Seoul St. Mary's Hospital, The

Catholic University of Korea,

222 Banpo-daero, Seocho-gu,

Seoul o6591, Korea

Tel: +82-2-2258-6043

Fax: +82-2-594-6043

E-mail: oncologykang@naver.com
Background/Aims: The outcome of local treatment for advanced non-small cell lung cancer (NSCLC) remains poor, with therapies such as induction chemotherapy (IC) yielding conflicting results. This study aimed to assess the clinicopathologic and prognostic significance of the excision repair cross-complementation group 1 (ERCC1), beclin-1, and glucose-regulated protein of molecular mass 78 $\left(\mathrm{GRP}_{7} 8\right)$ in patients with locally advanced NSCLC receiving docetaxel-platinum IC, along with efficacy and safety.

Methods: This is a retrospective observational cohort study. We reviewed medical records of 31 NSCLC patients receiving docetaxel-platinum IC, and conducted immunohistochemical staining of ERCC1, beclin-1, and GRP78.

Results: Response rate was $67.8 \%$ with 10.7 months of median relapse-free surviv$\mathrm{al}$ (RFS) and 23.1 months of median overall survival (OS), and no treatment-related death was reported. High expression of ERCC1, beclin-1, and GRP78 was identified in $67.7 \%, 87.1 \%$, and $67.7 \%$, respectively. Expression of ERCC1 and GRP78 did not reveal statistical significance in survival, whereas high beclin-1 expression revealed longer OS (7.6 months vs. 23.2 months; log-rank $p=0.024$ ). In multivariate analysis, histologic differentiation (hazard ratio [HR], 3.48; $p<0.001$ ), stage (HR, 8.5; $p=0.024)$, and adjuvant treatment (HR, $16.1 ; p=0.001)$ were related to RFS, and in OS, stage (HR, 5.4; $p=0.037$ ), adjuvant treatment (HR, 8.6; $p=0.004)$, and beclin-1 expression (HR, 8.2; $p=0.011$ ) were identified as significant prognostic factors.

Conclusions: Our findings suggest that high beclin-1 expression predicts longer survival in locally advanced NSCLC and docetaxel-platinum IC is a treatment option that deserves consideration.

Keywords: BECN1 protein, human; Carcinoma, non-small-cell lung; Induction chemotherapy

\section{INTRODUCTION}

Lung cancer is a leading cause of cancer deaths. Nonsmall cell lung cancer (NSCLC) accounts for many lung cancers, with up to one-third of patients with stage III of the disease. Despite advances in treatment modality, locally advanced NSCLC presents a therapeutic challenge. In terms of extent and size of the primary tumor, and 
nodal status, stage III NSCLC is a heterogeneous disease. The accepted treatment for locally advanced NSCLC is a multi-modal strategy including chemotherapy, radiation therapy, and surgery. However, with outcomes remaining poor, the most appropriate treatment remains controversial.

Induction chemotherapy (IC) in locally advanced NSCLC has revealed conflicting data [1,2]. IC may eradicate micrometastasis and assess tumor sensitivity to chemotherapy, and this information may be valuable for follow-up chemotherapy at the adjuvant and palliative stages. IC can diffuse more efficiently into the tumor, making it more advantageous than postradiation or surgery, and most importantly, lead to downstaging, thereby resulting in a higher percentage of curative resections. Conversely, there are drawbacks, including a delay in definitive local treatment and the potential for increased morbidity because of chemotherapy-induced toxicities. Therefore, many studies have focused on determining a more accurate prediction of tumor response, to select patients that would benefit most from IC.

The excision repair cross-complementation group 1 (ERCC1) enzyme plays a key role in the nucleotide excision repair system. It recognizes and removes platinum-induced DNA adducts; thus, its expression is associated with platinum resistance [3-5].

Hypoxia, hypoglycemia, and acidosis are main characteristics of tumor microenvironment, that lead to chronic endoplasmic reticulum (ER) stress. Glucose-regulated protein of molecular mass 78 (GRP78), a member of heat shock protein family, is a molecular chaperone in ER. Under ER stress, GRP78 activates a pro-survival pathway using an autophagy mechanism, and this leads to the protection and survival of cancer cells and chemoresistance $[6,7]$. Autophagy is a lysosomal pathway involved in cellular defense, with beclin-1 playing a key role in this mechanism [8]. Recently, several studies have been focusing on the role of autophagy in cancer. Autophagy has been suggested to be involved in taxane cytotoxicity, although this correlation is under investigation [9-13].

This purpose of this study was to assess the clinicopathologic and prognostic significance of ERCC1, beclin-1, and GRP7 8 in patients with locally advanced NSCLC receiving docetaxel-platinum IC, along with efficacy and safety of the treatment.

\section{METHODS}

\section{Patients and treatment}

We reviewed the medical records of NSCLC patients diagnosed and treated at Seoul St. Mary's Hospital January 2003 to June 2012. Patients that satisfied the following eligibility criteria were enrolled in this study: patients with histologically proven locally advanced NSCLC, an Eastern Cooperative Oncology Group (ECOG) performance status of $\leq 1$, available biopsied tissue acquired before IC, and those who received docetaxel-platinum IC for curative local treatment including surgery and concurrent chemoradiation treatment (CCRT). Cancer staging was evaluated according to the 7 th edition of the American Joint Committee on Cancer (AJCC). This study was approved by the Institutional Review Board (IRB) of the Catholic Medical Center (XC13TIMIooroK). IRB approved a waiver of informed consent. All patients were treated with $75 \mathrm{mg} / \mathrm{m}^{2}$ of docetaxel and $75 \mathrm{mg} / \mathrm{m}^{2}$ of cisplatin or carboplatin area under curve (AUC) 5 on day 1 every 3 weeks.

\section{Evaluation of tumor toxicity and response}

Toxicity was assessed according to the Common Terminology for Adverse Events version 3.0 (CTCAE v.3). Following completion of IC, tumor response was evaluated according to the Response Evaluation Criteria in Solid Tumors (RECIST 1.1).

\section{Immunohistochemical staining and scoring}

Immunohistochemical (IHC) staining was conducted using $4-\mu \mathrm{m}$ sections of paraffin-embedded tissue, biopsied for initial diagnosis. After deparaffinization in $100 \%$ xylene and rehydration over a series of decreasing concentrations of ethanol, using standard protocols, slides were subjected to antigen retrieval using the heat induced epitope retrieval method in $0.01 \mathrm{M}$ citrate buffer ( $\mathrm{pH}$ 6.0). Endogenous peroxidase activity was neutralized by immersion in $3 \%$ hydrogen peroxide in methanol for 10 minutes, followed by a $4{ }^{\circ} \mathrm{C}$ overnight incubation with mouse monoclonal anti-ERCC1 (1:200, GeneTex, Irvine, CA, USA), rabbit monoclonal anti-beclin-1 (1:200, Epitomics, Burlingame, CA, USA), and rabbit polyclonal anti-GRP78 (1:1,000, Abcam, Cambridge, UK). After washing, the sections were incubated with polymer-conjugated horseradish peroxidase for 
10 minutes at room temperature. After incubation, the sections were counterstained with hematoxylin. Two pathologists with no clinical information about the patients interpreted all immunostained slides. We scored expression levels by measuring the intensity of positive staining and graded as low and high.

\section{Statistical analysis}

Pearson chi-square test and Fisher exact test were used to assess the association between the expression of molecules (ERCC1, beclin-1, and GRP78) and clinicopathologic factors. Relapse-free survival (RFS) was defined as the time from the start of IC to relapse or death for patients dying without relapse. Overall survival (OS) was defined as the time between start of IC and death. We assessed the relationship between survival (RFS, OS) and the expression of molecules (ERCC1, beclin-1, and GRP78) using Kaplan-Meier methods. Univariate and multivariate Cox regression analyses were used to identify prognostic factors. We used the SPSS/PC+ version 18.0 (SPSS Inc., Chicago, IL, USA), and a p value of < 0.05 was considered statistically significant.

\section{RESULTS}

\section{Patient characteristics}

Thirty-one patients were enrolled in this study. Baseline characteristics are presented in Table 1. Mean age at diagnosis was 64 years (range, 43 to 75 ) and 27 patients $(87.1 \%)$ were men. ECOG performance status was reported as grade 1 in 25 patients (80.6\%), and 26 patients $(83.9 \%)$ were former or current smokers. Histology revealed squamous cell carcinoma in 20 patients (64.5\%), adenocarcinoma in 10 patients $(32.3 \%)$, and large cell carcinoma in one patient. Twenty-eight patients (90.3\%) had stage III disease, and three patients (9.7\%) had stage IIB disease. Twenty-eight patients (90.3\%) received docetaxel-cisplatin IC and three patients (9.7\%) received docetaxel-carboplatin. The median number of IC cycles was three (range, 2 to 4 ).

\section{Response, operability, and survival outcomes}

After two cycles of IC, three patients (9.7\%) demonstrated complete response, 18 patients $(58.1 \%)$ partial response, nine patients (29\%) stable disease, and one pa- tients (3.2\%) revealed progressive disease.

Following IC, 18 patients (58.1\%) underwent surgery, eight patients (25.8\%) received CCRT, two patients (6.5\%) received radiotherapy alone, two patients $(6.5 \%)$ were treated with second-line chemotherapy or best supportive care, and one patient was lost to follow-up. Of 18 patients undergoing surgical resection, 15 patients (83.3\%) achieved Ro resection, and 12 patients $(66.7 \%)$ revealed pathologic downstaging. Two patients died within 30 days postsurgery (massive hemoptysis and sepsis), and one patient died of radiation pneumonitis after defini-

Table 1. Patient characteristics $(\mathbf{n}=31)$

\begin{tabular}{|c|c|}
\hline Characteristic & Value \\
\hline Male sex & $27(87.1)$ \\
\hline Age, yr & $64.4 \pm 7.1$ \\
\hline \multicolumn{2}{|l|}{ Performance status } \\
\hline o & $6(19.4)$ \\
\hline 1 & $25(80.6)$ \\
\hline \multicolumn{2}{|l|}{ Smoking status } \\
\hline Never-smoker & $5(16.1)$ \\
\hline Former-smoker & $14(45 \cdot 2)$ \\
\hline Current-smoker & $12(38.7)$ \\
\hline \multicolumn{2}{|l|}{ Pathology } \\
\hline Squamous cell & $20(64.5)$ \\
\hline Adenocarcinoma & $10(32.3)$ \\
\hline Large cell & $1(3.2)$ \\
\hline \multicolumn{2}{|l|}{ Histologic differentiation } \\
\hline Moderate & $20(64.5)$ \\
\hline Poor & $11(35 \cdot 5)$ \\
\hline \multicolumn{2}{|l|}{ Stage } \\
\hline IIB & $3(9.7)$ \\
\hline IIIA & $21(67.7)$ \\
\hline IIIB & $7(22.6)$ \\
\hline \multicolumn{2}{|l|}{ Regimen } \\
\hline Docetaxel-cisplatin & $28(90.3)$ \\
\hline Docetaxel-carboplatin & $3(9.7)$ \\
\hline \multicolumn{2}{|l|}{ No. of IC cycles } \\
\hline 2 & $15(48.4)$ \\
\hline 3 & $15(48.4)$ \\
\hline 4 & $1(3.2)$ \\
\hline RDI & $89.4 \pm 11.7$ \\
\hline
\end{tabular}

Values are presented as number (\%) or mean \pm SD.

IC, induction chemotherapy; RDI, relative dose intensity. 
Table 2. Toxicities of docetaxel-platinum induction chemotherapy in locally advanced non-small cell lung cancer

\begin{tabular}{lcc}
\hline & All grades & Grade 3-4 \\
\hline Hematologic toxicities & & \\
\hline Neutropenia & $23(74.2)$ & $21(67.7)$ \\
\hline Anemia & $11(35.5)$ & $1(3.2)$ \\
\hline Thrombocytopenia & $5(16.1)$ & $1(3.2)$ \\
\hline Non-hematologic toxicities & & \\
\hline Asthenia & $27(87.1)$ & $1(3.2)$ \\
\hline Myalgia & $15(48.2)$ & 0 \\
\hline Anorexia & $26(83.9)$ & $1(3.2)$ \\
\hline Nausea & $18(58.1)$ & $2(6.5)$ \\
\hline Vomiting & $10(32.3)$ & $2(6.5)$ \\
\hline Diarrhea & $11(35.5)$ & $2(6.5)$ \\
\hline Constipation & $4(12.9)$ & 0 \\
\hline Alopecia & $22(71)$ & 0 \\
\hline Mucositis & $12(38.7)$ & $2(6.5)$ \\
\hline Neutopathy & $6(19.4)$ & 0 \\
\hline Rash & $5(16.1)$ & 0 \\
\hline
\end{tabular}

Values are presented as number (\%).

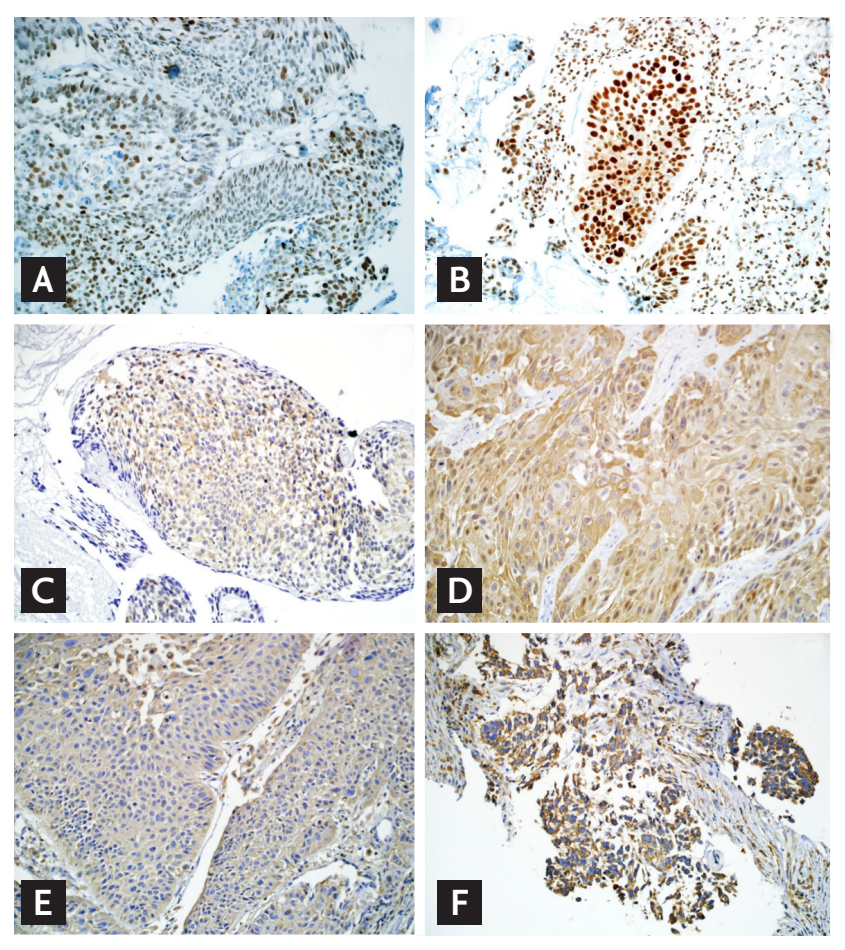

Figure 1. Expression by immunohistochemistry ( $\times 200)$. (A) Low excision repair cross-complementation group 1 (ERCC1). (B) High ERCC1. (C) Low beclin-1. (D) High beclin-1. (E) Low glucose-regulated protein of molecular mass 78 (GRP78). (F) High GRP78. tive CCRT. Adjuvant CCRT was administered to six patients after surgical resection and adjuvant chemotherapy to nine patients.

Median follow-up duration was 18.8 months (range, 3 to 130), and median RFS and OS was 10.7 months (95\% confidence interval [CI], 5.5 to 15.8 ) and 23.1 months (95\% CI, 15.1 to 31), respectively.

\section{Toxicities}

Over the course of chemotherapy, asthenia, anorexia, neutropenia, alopecia, nausea, and myalgia were frequently observed (Table 2). However, all toxicities were transient and were managed with supportive care. Treatment-related death did not occur. The most common grade $3 / 4$ toxicity was neutropenia $(67.7 \%)$ and febrile neutropenia occurred in a patient. Grade 3/4 neutropenia was managed with granulocyte-colony stimulating factor (G-CSF), with or without antibiotics. Most of the patients recovered from neutropenia within 3 days and we did not observe delay of chemotherapy from prolonged toxicities of $>1$ week. The mean duration from last chemotherapy treatment to surgery was $1.1 \pm 0.3$ months (range, 20 to 66 days).

\section{Expression of ERCC 1 , Beclin-1, and GRP78}

High expression of ERCC1, beclin-1, and GRP78 were identified in 21 patients $(67.7 \%), 27$ patients $(87.1 \%)$, and 21 patients (67.7\%), respectively (Fig. 1). High ERCC1 expression was found frequently in low $\mathrm{T}$ staging $(\mathrm{T} 1,2)$ compared to high $\mathrm{T}$ staging $\left(\mathrm{T}_{3}, 4\right)$ with a relative risk of $2(p=0.021)$. Correlations between expression of the molecules and other clinicopathologic features or tumor response were not statistically significant (Table 3). In addition, relationships between expression of ERCC1, beclin-1, and GRP78 were not identified (Table 4).

\section{Predictive and prognostic role of ERCC 1 , Beclin-1, and GRP78}

In the Kaplan-Meier analysis of RFS, the expression of ERCC1, beclin-1, and GRP78 did not reveal statistical significance (median RFS in patients with low vs. high expression; ERCC1: 6.9 months vs. 10.7 months, log-rank $p=0.775$; beclin-1: 6.3 months vs. 10.7 months, log-rank $p=0.185$; GRP78: 6.9 months vs. 10.7 months, $\log$-rank $p=0.314)$. In the analysis of OS, patients with high expression of beclin-1 showed longer OS (median 
Table 3. Correlation between expression of molecules (ERCC1, beclin-1, and GRP78) and clinicopathologic characteristics

\begin{tabular}{|c|c|c|c|c|c|c|}
\hline & \multicolumn{2}{|c|}{ ERCC1 } & \multicolumn{2}{|c|}{ Beclin-1 } & \multicolumn{2}{|c|}{$\mathrm{GRP}_{7} 8$} \\
\hline & High & $p$ value & High & $p$ value & High & $p$ value \\
\hline All cases & 21 & & 27 & & 21 & \\
\hline \multicolumn{7}{|l|}{ Smoking } \\
\hline Never-smoker:smoker & $3: 18$ & 1 & $5: 22$ & 1 & $5: 16$ & 0.147 \\
\hline \multicolumn{7}{|l|}{ Pathology } \\
\hline SqCC:non-SqCC & $14: 7$ & 1 & $20: 11$ & 1 & $12: 9$ & 0.262 \\
\hline \multicolumn{7}{|l|}{ Histologic differentiation } \\
\hline MD:PD & $14: 7$ & 1 & $19: 8$ & 0.115 & $13: 8$ & 1 \\
\hline \multicolumn{7}{|l|}{ Stage } \\
\hline $\mathrm{T}_{1}, 2: \mathrm{T}_{3}, 4$ & $16: 5$ & 0.021 & $19: 12$ & 0.619 & $14: 7$ & 0.447 \\
\hline $\mathrm{No}: \mathrm{N}_{1}, 2,3$ & $3: 18$ & 0.739 & $4: 23$ & 1 & $4: 17$ & 0.277 \\
\hline IIB, IIIA:IIIB & $17: 4$ & 0.652 & $21: 6$ & 1 & $16: 5$ & 1 \\
\hline \multicolumn{7}{|l|}{ Tumor response } \\
\hline $\mathrm{CR}+\mathrm{PR}: \mathrm{SD}+\mathrm{PD}$ & $15: 6$ & 0.685 & $18: 9$ & 1 & $14: 7$ & 1 \\
\hline
\end{tabular}

Values are presented as number.

ERCC1, excision repair cross-complementation group 1; GRP78, glucose-regulated protein of molecular mass 78; SqCC, squamous cell carcinoma; $\mathrm{MD}$, moderately differentiated; $\mathrm{PD}$, poorly differentiated; $\mathrm{CR}$, complete response; PR, partial response.

Table 4. Correlations between expression of ERCC1, beclin-1, and GRP 78

\begin{tabular}{|c|c|c|c|c|c|c|}
\hline & \multicolumn{3}{|c|}{ ERCC1 } & \multicolumn{3}{|c|}{ GRP78 } \\
\hline & High & Low & $p$ value & High & Low & $p$ value \\
\hline \multicolumn{7}{|l|}{ Beclin-1 } \\
\hline High & 18 & 9 & 1 & 18 & 9 & 1 \\
\hline Low & 3 & 1 & & 3 & 1 & \\
\hline \multicolumn{7}{|l|}{$\mathrm{GRP}_{78}$} \\
\hline High & 16 & 5 & 0.222 & & & \\
\hline Low & 5 & 5 & & & & \\
\hline
\end{tabular}

ERCC1, excision repair cross-complementation group 1; GRP78, glucose-regulated protein of molecular mass 78 .

Table 5. Prognostic factors for survival by multivariate analysis

\begin{tabular}{|c|c|c|c|c|}
\hline \multirow{2}{*}{ Factor } & \multicolumn{2}{|c|}{ RFS } & \multicolumn{2}{|c|}{ OS } \\
\hline & HR & $p$ value & HR & $p$ value \\
\hline Differentiation (PD vs. MD) & 34.8 & $<0.001$ & & \\
\hline Stage (IIIB vs. IIB, IIIA) & 8.5 & 0.118 & $5 \cdot 4$ & 0.037 \\
\hline \multicolumn{5}{|l|}{ Nodal stage (N1, 2, 3 vs. No) } \\
\hline \multicolumn{5}{|c|}{ Definitive treatment (CCRT vs. surgery) } \\
\hline Adjuvant treatment (no vs. yes) & 16.1 & 0.001 & 8.6 & 0.004 \\
\hline Beclin-1 (low vs. high) & & & 8.2 & 0.011 \\
\hline
\end{tabular}

RFS, relapse-free survival; OS, overall survival; HR, hazard ratio; PD, poorly differentiated; MD, moderately differentiated; CCRT, concurrent chemoradiation. 


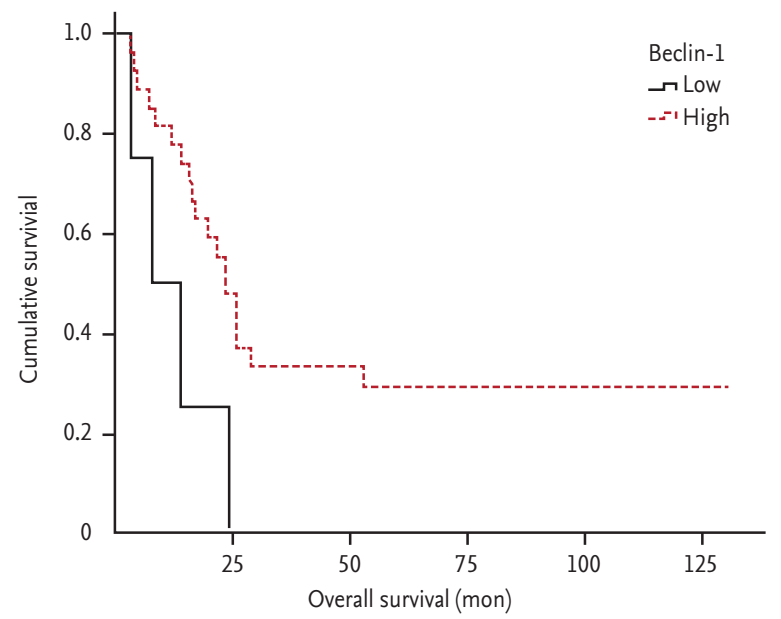

Figure 2. Overall survival of patients with non-small cell lung cancer according to beclin-1 expression.

OS in patients with low vs. high expression; ERCC1: 23.1 months vs. 19.5 months, log-rank $p=0.647$; beclin-1: 7.6 months vs. 23.2 months, log-rank $p=0.024$; GRP78: 19.5 months vs. 23.2 months, log-rank $p=0.547$ ) (Fig. 2). Univariate Cox regression analysis revealed that histologic differentiation (moderate vs. poor; hazard ratio [HR], 17.8 and $p<0.001$ ) was significantly related to RFS and beclin-1 expression (low vs. high; HR, 3.6 and $p=0.012$ ) and adjuvant treatment after definitive treatment (no vs. yes; HR, 4.4 and $p=0.035$ ) were related to OS. Multivariate analysis conducted with factors exhibiting $p<0.2$ at univariate analysis (histologic differentiation, stage, nodal stage, type of definitive treatment, additional adjuvant treatment, and expression of beclin-1). Identified significant factors were histologic differentiation, stage, and adjuvant treatment for PFS, and stage, adjuvant treatment and expression of beclin-1 for OS (Table 5).

\section{DISCUSSION}

This study was conducted to find predictive and prognostic biomarkers in patients with locally advanced NSCLC receiving docetaxel-platinum IC, in company with efficacy and safety of IC. Our results suggested beclin-1 as a prognostic biomarker, and IC as a safe and effective treatment option.

In our study, patients with high expression of beclin-1 revealed longer OS, while significant predictive role of its expression in terms of IC such as RFS or tumor re- sponse was not identified. However, high beclin-1 expression revealed a tendency towards longer RFS. Since only four patients $(12.9 \%)$ revealed low expression of beclin-1, further study with an expanded cohort and standardizing method of beclin-1 assessment is needed. Autophagy maintains intracellular homeostasis which has conflicting roles in cancer cells, anti-tumors and pro-tumors. Beclin-1 is essential for autophagosome formation, and in human cancer, its downregulation and tumor suppressive role have been reported [12,1416]. In cervical and duodenal cancer, low beclin-1 expression was associated with poor prognosis and in cervical cancer, the expression of beclin-1 in tumor tissue was lower compared to adjacent normal tissue [15,16]. In breast cancer, autophagy enhanced paclitaxel-induced cell death and autophagy-related markers are downregulated in docetaxel-resistant breast cancer cell lines [17]. Meanwhile, by contrast with aforementioned studies and our results, in endometrial and pancreatic cancer, high beclin-1 expression was associated with poor prognosis, suggesting a pro-tumor role of beclin-1 [18,19]. In NSCLC, autophagy and beclin-1 have been studied as well and conflicting results were reported. Wang et al. [12] and Kim et al. [20] reported consistent results with our study that low beclin-1 expression was associated with aggressive behavior and poor prognosis. Whereas several preclinical studies with NSCLC cell lines reported different results supporting pro-tumor role of autophagy, revealing contribution of autophagy to taxane resistance. In this context, autophagy was proposed as a therapeutic target to overcome chemoresistance $[11,13]$. In our study and the abovementioned studies, Wang et al. [12] and Kim et al. [20] reported a majority of patients had squamous cell histology (64.5\%, 51.9\%, and 51.9\%, respectively), while the NSCLC cell lines in the preclinical studies revealing a pro-tumor role of autophagy were all adenocarcinoma cell lines [11-13]. Hence, in NSCLC, the role of autophagy may be different according to histology. The different roles of autophagy according to histologic subtype were not identified in our study, so further study is needed in an expanded cohort and preclinical setting with squamous histology cell lines. Beclin-1 expression did not have a significant predictive role in terms of IC such as RFS or tumor response; however, high beclin-1 expression revealed a tendency towards longer RFS. 
In our study, high expression of GRP78 revealed longer RFS and OS compared to low expression group with no statistical significance. Emerging data indicates that ER stress and autophagy are involved in human cancer, specifically in carcinogenesis, disease aggressiveness, metastasis, and sensitivity/resistance to anti-tumor treatment [6-10]. Kim et al. [20] reported that expression of GRP78 and beclin-1 in NSCLC correlates with low tumor stage and longer survival, and that their expressions correlated with each other. Conversely, Sun et al. [21] reported that GRP78 mRNA expression was significantly higher (71.9\%) in stage III/IV NSCLC, like our result of $71.7 \%$. Additionally, in a small interfering RNA experiment, they have revealed that $\mathrm{GRP}_{7} 8$ promotes metastasis and anti-apoptosis.

Besides the undefined roles of autophagy and ER stress in NSCLC, several factors may contribute to results with lack of statistical significance $[9,10,17]$. First, we focused on locally advanced disease, mainly IIIB. We had limited sample numbers $(n=31)$, and there is concern in serving biopsied tissue as a representative sample for whole tumor.

Being not in accord with previous studies, neither predictive nor prognostic role of ERCC1 was identified in our study. A recent study suggested that IHC analysis with commercially available $8 \mathrm{~F} 1$ antibodies did not specifically detect the unique functional ERCC1 isoform, thereby limiting its use in the clinic towards therapeutic decision making [22].

In the present study, the response rate of docetaxel-platinum IC was higher (67.8\%) than those of previous studies employing paclitaxel-carboplatin IC (40\% to $55 \%$ ), and median OS (23.1 months) was similar to those of previous reports (OS, 16 to 29 months). Considering that about a quarter of patients in this study had stage IIIB disease, while previous reports focused on stage IIIA disease, the result of this study is notable [23-25]. In terms of toxicities, the present study revealed a higher incidence of grade $3 / 4$ neutropenia $(78.4 \%)$ than that with paclitaxel-carboplatin or gemcitabine-cisplatin IC [23,25]. Ethnic differences of pharmacogenomics in docetaxel metabolism may contribute to the serious hematologic toxicities. However, these were transient and successfully managed in all patients using G-CSF and isolation care. In addition, these toxicities did not lead to delay in the follow-up definitive local treatment, and we ob- served no treatment-related death. The addition of adjuvant treatment after definitive treatment was identified as a viable prognostic factor in RFS and OS. Hence, IC followed by surgery and adjuvant treatment could be an optimal sequence. And the addition of immunotherapy and detection of cell free tumor DNA after definitive treatment might play roles in the treatment of locally advanced NSCLC [26].

Our findings suggested beclin-1 expression as a favorable prognostic biomarker in locally advanced NSCLC. Further study is required to validate biomarkers related to autophagy and ER stress in NSCLC.

\section{KEY MESSAGE}

1. Our study suggested the possibility of beclin-1 expression as a favorable prognostic marker in locally advanced non-small cell lung cancer (NSCLC) receiving docetaxel-platinum induction chemotherapy.

2. Further extended study is required to validate biomarkers related to autophagy and endoplasmic reticulum stress in NSCLC.

\section{Conflict of interest}

No potential conflict of interest relevant to this article was reported.

\section{REFERENCES}

1. Gilligan D, Nicolson M, Smith I, et al. Preoperative chemotherapy in patients with resectable non-small cell lung cancer: results of the MRC LU22/NVALT 2/EORTC 08012 multicentre randomised trial and update of systematic review. Lancet 2007;369:1929-1937.

2. Song WA, Zhou NK, Wang W, et al. Survival benefit of neoadjuvant chemotherapy in non-small cell lung cancer: an updated meta-analysis of 13 randomized control trials. J Thorac Oncol 2010;5:510-516.

3. Besse B, Olaussen KA, Soria JC. ERCC1 and RRMı: ready for prime time? J Clin Oncol 2013;31:1050-1060.

4. Olaussen KA, Dunant A, Fouret P, et al. DNA repair by ERCC1 in non-small-cell lung cancer and cisplatin-based adjuvant chemotherapy. N Engl J Med 2006;355:983-991. 
5. Wachters FM, Wong LS, Timens W, Kampinga HH, Groen HJ. ERCC1, hRad51, and BRCA1 protein expression in relation to tumour response and survival of stage III/IV NSCLC patients treated with chemotherapy. Lung Cancer 2005;50:211-219.

6. Lee AS. GRP78 induction in cancer: therapeutic and prognostic implications. Cancer Res 2007;67:3496-3499.

7. Pyrko P, Schonthal AH, Hofman FM, Chen TC, Lee AS. The unfolded protein response regulator GRP78/BiP as a novel target for increasing chemosensitivity in malignant gliomas. Cancer Res 2007;67:9809-9816.

8. Wirawan E, Lippens S, Vanden Berghe T, et al. Beclinı: a role in membrane dynamics and beyond. Autophagy 2012;8:6-17.

9. White E, DiPaola RS. The double-edged sword of autophagy modulation in cancer. Clin Cancer Res 2009;15:53085316.

10. Morselli E, Galluzzi L, Kepp O, et al. Anti- and pro-tumor functions of autophagy. Biochim Biophys Acta 2009;1793:1524-1532.

11. Chen K, Shi W. Autophagy regulates resistance of nonsmall cell lung cancer cells to paclitaxel. Tumour Biol 2016;37:10539-10544.

12. Wang X, Du Z, Li L, Shi M, Yu Y. Beclin 1 and p62 expression in non-small cell lung cancer: relation with malignant behaviors and clinical outcome. Int J Clin Exp Pathol 2015;8:10644-10652.

13. Pan B, Chen D, Huang J, et al. HMGB1-mediated autophagy promotes docetaxel resistance in human lung adenocarcinoma. Mol Cancer 2014;13:165.

14. Aita VM, Liang XH, Murty VV, et al. Cloning and genomic organization of beclin 1, a candidate tumor suppressor gene on chromosome 17q21. Genomics 1999;59:59-65.

15. Zhu W, Pan X, Li F, Zhang Y, Lu X. Expression of Beclin 1 and LC $_{3}$ in FIGO stage I-II cervical squamous cell carcinoma and relationship to survival. Tumour Biol 2012;33:1653-1659.

16. Wu XY, Chen J, Cao QH, et al. Beclin 1 activation enhances chemosensitivity and predicts a favorable outcome for primary duodenal adenocarcinoma. Tumour Biol 2013;34:713-722.
17. Veldhoen RA, Banman SL, Hemmerling DR, et al. The chemotherapeutic agent paclitaxel inhibits autophagy through two distinct mechanisms that regulate apoptosis. Oncogene 2013;32:736-746.

18. Ko YH, Cho YS, Won HS, et al. Prognostic significance of autophagy-related protein expression in resected pancreatic ductal adenocarcinoma. Pancreas 2013;42:829-835.

19. Giatromanolaki A, Koukourakis MI, Koutsopoulos A, Chloropoulou P, Liberis V, Sivridis E. High Beclin 1 expression defines a poor prognosis in endometrial adenocarcinomas. Gynecol Oncol 2011;123:147-151.

20. Kim KM, Yu TK, Chu HH, et al. Expression of ER stress and autophagy-related molecules in human non-small cell lung cancer and premalignant lesions. Int J Cancer 2012;131:Е362-E370.

21. Sun Q, Hua J, Wang Q, et al. Expressions of GRP78 and Bax associate with differentiation, metastasis, and apoptosis in non-small cell lung cancer. Mol Biol Rep 2012;39:67536761.

22. Friboulet L, Olaussen KA, Pignon JP, et al. ERCC1 isoform expression and DNA repair in non-small-cell lung cancer. N Engl J Med 2013;368:1101-1110.

23. Felip E, Rosell R, Maestre JA, et al. Preoperative chemotherapy plus surgery versus surgery plus adjuvant chemotherapy versus surgery alone in early-stage non-small-cell lung cancer. J Clin Oncol 2010;28:3138-3145.

24. Pisters KM, Vallieres E, Crowley JJ, et al. Surgery with or without preoperative paclitaxel and carboplatin in early-stage non-small-cell lung cancer: Southwest Oncology Group Trial S99oo, an intergroup, randomized, phase III trial. J Clin Oncol 2010;28:1843-1849.

25. Scagliotti GV, Pastorino U, Vansteenkiste JF, et al. Randomized phase III study of surgery alone or surgery plus preoperative cisplatin and gemcitabine in stages IB to IIIA non-small-cell lung cancer. J Clin Oncol 2012;30:172178.

26. Berman AT, Simone CB 2nd. Immunotherapy in locally-advanced non-small cell lung cancer: releasing the brakes on consolidation? Transl Lung Cancer Res 2016;5:138-142. 